\title{
Circadian rhythms, athletic performance, and jet lag
}

\author{
Roberto Manfredini, Fabio Manfredini, Carmelo Fersini, Francesco Conconi
}

\begin{abstract}
Rapid air travel across several time zones exposes the traveller to a shift in his/her internal biological clock. The result is a transient desynchronisation of the circadian rhythm, called jet lag, lasting until the rhythm is rephased to the new environmental conditions. The most commonly experienced symptoms are sleep disorders, difficulties with concentrating, irritability, depression, fatigue, disorientation, loss of appetite, and gastrointestinal disturbance. Apart from the decrements in mental and physical performance directly consequent on such symptoms, competitive athletes are also exposed to the additional negative consequences of a shift from the optimal circadian window of performance. A brief summary of the possible negative effects of jet lag on athletic performance and potentially alleviating strategies is given. (Br J Sports Med 1998;32:101-106)
\end{abstract}

Keywords: athletic performance; jet lag; chronobiology; circadian rhythms

Chronobiology can be defined as the study of rhythmic patterns in biological phenomena. Oscillatory fluctuations, called biological rhythms, occur in cells, tissues, organs, and more complex control systems. They are endogenous, arise within the organism, and persist under constant environmental conditions. Although they may show a wide range of periods, circadian rhythms (from the Latin circa diem meaning about 24 hours) are the most extensively investigated. Rhythms are not imposed by the environment, but can be adjusted by exogenous synchronising cues-for example, alternation of light and dark. However, the night-day cycle has progressively lost some of its importance because of the invention of artificial light, which allows work by night. Together with shift work, the most important cause of internal desynchronisation is the rapid crossing of several time zones, called jet lag. ${ }^{1}$ Travel across several time zones has become a necessity for international sport competitors, with possible impairments of performance until adjustment to the local time is achieved.
Both the 1998 winter and 2000 summer Olympic Games (scheduled to take place in respectively Japan and Australia) will require the crossing of several time zones, especially for European teams, and chronobiology may be a useful tool for defining the optimal time for training and competing, thereby increasing personal performance and alleviating possible jet lag induced disturbances. While waiting for the final results of the JAP study, a project targeted at the Nagano 1998 winter Olympic Games, the aim of this paper is to review briefly current knowledge on the possible effects of jet lag on physical performance in athletes and potentially alleviating strategies.

\section{Jet lag}

Rapid air travel across several time zones exposes the traveller to a shift in the internal biological clock. The result is a transient desynchronisation of rhythm, lasting until the biological rhythms adjust to the new environmental conditions. The subjective symptoms usually associated include sleep disorders, difficulties with concentrating, irritability, depression, disorientation, distorted estimation of time, space, and distance, lightheadedness, loss of appetite, and gastrointestinal disturbances. ${ }^{12}$ Air crews report sleep disturbances in about $60-70 \%$ of cases on the first night after crossing a time zone, which is reduced to $30 \%$ on the third day ${ }^{3}$; loss of sleep amounts to as much as five or six hours per night flight. ${ }^{4}$ Moreover, female flight attendants experience more irregular menstrual cycles. ${ }^{5}$ It is well known that westward flights (characterised by a phase delay) are followed by faster recovery and resynchronisation than eastward flights (phase advance), and sleep quality decreases particularly after eastward flights. ${ }^{6}$ Klein and Wegmann $^{7}$ calculated that three days were needed to resynchronise psychomotor performance rhythms after a westward flight from Germany to the United States, whereas eight days were required for the reverse direction. After a time zone crossing of several hours you can recover day by day. After a westward flight the mean re-entrainment shift rate is about $92 / \mathrm{min} /$ day, and only $57 / \mathrm{min} /$ day after eastward flights. ${ }^{8}$ However, there is a considerable variation in the rate of readaptation between individuals for a given rhythm and between rhythms in a given individual. About $30 \%$ of transmeridian travellers have little or no 
difficulty adjusting to the temporary circadian desynchronisation and are asymptomatic, but another $30 \%$ do not adjust well at all. ${ }^{9}$ Symptoms are commonly reported during the 48 hours immediately after a flight, and the more time zones that have been traversed, the longer is the period of recovery, although the relationship is not linear. ${ }^{10}$ Individual factors associated with widely differing rates of rhythmic readaptation are: direction of flight, synchroniser strength, rhythm stability, personality chronotype, rhythm amplitude, behavioural traits, and sleep habits. ${ }^{9}$ Thus, for example, evening type people are less sensitive than morning types, subjects with low amplitudes in body temperature seem to be less resistant to phase shift than individuals with large circadian amplitudes, ${ }^{1}$ and older travellers suffer more than younger ones. ${ }^{11}$ It has also been reported that re-entrainment after transmeridian flights is more rapid in summer than in winter, possibly because the longer day permits greater exposure to natural daylight. ${ }^{12}$

Jet lag and athletic performance

Some aspects of chronobiology are important to physical performance-for example, the normal cyclic variation observed in the physiological mechanisms that contribute to overall athletic performance, the effect of abnormal rhythmicity or desynchronisation on athletic performance, and the alleviating counter measures.

HUMAN CIRCADIAN RHYTHMS AT REST

Body temperature

Body temperature starts to rise before waking, reaches a peak at about 1800 hours, then falls during sleep, with a nadir at around 0400 hours; an amplitude of $0.4-0.5^{\circ} \mathrm{C}$ is seen in young adults. ${ }^{13}$ The circadian rhythm of body temperature is mainly the result of fluctuations in heat loss mechanisms rather than heat production, ${ }^{13}$ maybe with the intervention of noradrenergic peaks. ${ }^{14}$

Heart rate, blood pressure, and ventilation

Heart rate oscillates during the day, with a peak at around 1500 hours and an amplitude of $5-15 \% .{ }^{15}$ An analogous pattern is demonstrable for stroke volume, cardiac output, blood flow, and blood pressure. ${ }^{16}$ Both heart rate and blood pressure are greatly influenced by exogenous factors, such as sleep, posture, diet, and activity, ${ }^{17}$ although it has been shown that the temporal organisation of blood pressure is mainly controlled by neuroendocrine mechanisms, coupled to either sleep or an endogenous pacemaker which is usually predominantly (although not solely) circadian. ${ }^{18}$

\section{Gastrointestinal and urinary function}

Circadian rhythms are associated with several gastrointestinal functions - for example, motility patterns, enzyme activity, and acid secretion. ${ }^{19}$ Gastric emptying rates, for example, are over $50 \%$ slower for evening than morning meals. ${ }^{20}$ As for urinary function, urinary electrolytes show an afternoon peak, ${ }^{21}$ and urinary $\mathrm{pH}$ is lower during sleep, and higher in the morning. ${ }^{22}$
Chronotype

An important factor is personality "chronotype" - that is, whether one is a morning person ("lark"), who gets up and goes to bed early, or an evening person ("owl"), who wakes up and retires late. There is a difference of about 65 minutes in the peaks of body temperature rhythm between morning and evening types, and morning types secrete significantly more adrenaline in the morning than do evening types. Furthermore, the timing of mood and activity rhythms differs by several hours between distinct morning and evening types. ${ }^{23}$

CIRCADIAN VARIATIONS IN SPORTS PERFORMANCE Interested readers should read the excellent recent review by Atkinson and Reilly. ${ }^{24} \mathrm{We}$ report here a few briefly summarised data from it. Although considerations such as environmental influences, temperature, meteorological conditions, and scheduled times of events make generalisation difficult, most components of sports performance exhibit a rhythmic variation during the day, with a peak in the early evening. This time of day is characterised by peaks in reaction time, ${ }^{25}$ isometric hand grip strength, ${ }^{26}$ elbow flexion strength, ${ }^{27}$ back strength, ${ }^{28}$ total work performed in high intensity constant work rate exercise, ${ }^{29}$ and lactate production, ${ }^{30}$ and lowest levels of joint stiffness ${ }^{31}$ and pain perception. ${ }^{32}$ When subjects are free to chose their submaximal work rate during exercise of less than 40 minutes duration, higher work rates are achieved in the early evening without any change in the perception of effort. ${ }^{33}$ Moreover, in young adults, the mean work rate over 80 minutes of exercise was found to be higher in the afternoon than in the morning. ${ }^{34}$ Improvements in muscle strength after training sessions scheduled in the evening have been found to be $20 \%$ higher than those after training carried out in the morning. ${ }^{35}$ Long term memory recall, in which data must be retained for one week or longer, is $8 \%$ greater when the material is presented at 1500 hours than at 0900 hours. ${ }^{36}$ The implications are important for the timing of coaching instructions and strategy, since the $8 \%$ difference in memory retention is similar to the performance decrement induced when sleep is restricted to three hours. When individual differences in performances were controlled for, a significant early evening peak was found for length of jump ${ }^{37}$ and vertical jumping performance. ${ }^{38}$ Swimming performance shows an evening peak too, ${ }^{39}$ having rhythm amplitudes of 11 to $14 \%$ of the 24 hour mean values. The circadian variation in swimming performance is greater than the effect of obtaining only three hours of sleep for three successive nights. ${ }^{40}$ It has to be stressed that there is an inverse relation between speed and accuracy in a simple repetitive test, ${ }^{41}$ with accuracy being worse in the early evening. Remembering that other aspects of performance-for example, mental arithmetic and short term memory-peak in the early morning rather than evening hours, ${ }^{42}$ it is possible that times of day other than the evening may be better for sports that demand accuracy, for competitive strategies, and for delivery and recall of coaching instructions. 
DESYNCHRONISATION AND ATHLETIC PERFORMANCE

A poor competitive performance may result when an athlete does not take into consideration his or her circadian performance profile, since an athletic task undertaken several hours before or after the circadian peak "window" will potentially be performed with less than optimal efficiency. Taking circadian rhythms into consideration can produce major benefits in tasks involving endurance, mental function, physical strength, and others. Selecting the best circadian time can result in as much as a $10 \%$ increase in athletic performance. A $10 \%$ decrement in peak performance can be compared with a performance after less than three hours of sleep, after drinking the legal limit of alcohol, ${ }^{43}$ or after taking barbiturates. ${ }^{44}$

Jet lag may cause a shift in the circadian peak window. It has been reported that the performances of travelling American football teams depend on how closely the game time corresponds to the usual afternoon peaks in performance. ${ }^{45}$ West coast teams, in fact, appear to be at an advantage over East and Central teams for night games.

Although it is somewhat difficult to investigate the negative effects of jet lag in competitive athletes, because of the masking of negative effects of stress, fatigue caused by the flight itself, and the forced situation of competing in a "different" environment, a very interesting contribution has been recently provided by Recht and co-workers. ${ }^{46}$ They analysed records for three complete seasons (1991-1993) of the 19 North American major league baseball teams based in cities of the Eastern and Pacific time zones. These teams had significantly more victories when playing at home than away $(54 \% v 46 \%)$, and it was found that the probability of winning depended on whether the visiting team had just travelled eastward. The home team could, in fact, expect to score 1.24 more runs than usual when the visitors had just completed an eastward journey. The authors concluded that, although there are many factors, one critical component of the "home field" advantage involved previous transcontinental travel by the visiting team within the preceding two days, but only in the case of an eastward direction. In accordance with such results, previous studies on a series of surveys on the scores of international games held in Japan and their relation to the length of stay of the visiting team ${ }^{47}$ showed an increase in performance level (as judged by the outcome of their matches) in parallel with adjustment to the new time zone.

A recent study by Reilly et $a l^{48}$ showed that, in British Olympic squad members, several performance measures-for example, leg and back strength, choice reaction time, and subjective jet lag symptoms - were impaired for five days after a trip from the United Kingdom to Florida. The first to be re-established was the sleep-wake cycle, then body temperature and absence of jet lag symptoms before the complete normalisation of performance measures rhythms.
JET LAG ALLEVIATING MEASURES

Several measures have been suggested ro reduce the negative effects of circadian rhythm desynchronisation, although treatment of shift workers, competitive athletes, and simple international travellers is quite different. The measures include preadaptation, meal timing and composition, phototherapy, and chronobiotic drugs.

\section{Preadaptation}

Theoretically, the negative effects of jet lag can be reduced by changing bedtime for several days before a transcontinental journey, remembering that sleep changes should correspond to the direction of travel (eastward or westward). Adaptation is disrupted by behaviour that anchors the circadian rhythms to the previous phase-for example, taking prolonged naps at the new destination. ${ }^{49}$ To lock the circadian rhythms to home time, at least four hours sleep taken within the window of normal sleep in the home time zone are needed. ${ }^{9}$ However, in practice, preadaptation is superfluous when travelling west, arriving late, and only a few hours phase advance can be recommended before travelling east. ${ }^{50}$ Moreover, because of the difficulty of manipulating other synchronising factors (light, social constraints), preadjusting the sleep-wake cycle is largely ineffective. ${ }^{51}$

\section{Timing and composition of meals}

Certain food constituents seem to have effects on rhythm adjustment. A high carbohydrate low protein meal, facilitating brain uptake of tryptophan and its conversion to serotonin, may induce drowsiness and sleep. On the other hand, a high protein low carbohydrate meal, which enhances tyrosine uptake and conversion to adrenaline, increases arousal levels. ${ }^{52}$ Moreover, programmed use of theophylline and caffeine can speed up rhythm readaptation, and help raise arousal levels in the morning. However, although studies on military personnel consuming such diets reported reduced sleep disturbance and less subjective feelings of fatigue in the days immediately after a transmeridian flight compared with controls, ${ }^{2}$ a clear link between diet and jet lag has not been formally established.

\section{Phototherapy}

When an individual's circadian phase is known, it is possible to act on the central clock mechanism, advancing or delaying the clock itself. Exposure to bright light, with specified intensity, duration, and timing, may advance or delay the phase of some human circadian rhythms. ${ }^{53}$ Most available studies have used jet lag simulations, so the subjects are not exposed to the full complement of time cues normally encountered by transmeridian travellers; this has been reviewed by Boulos et al. ${ }^{54}$ Two studies have investigated the effects of light treatment on post-flight sleep patterns. In the former, ${ }^{55} 19$ subjects returning to the United States from Oriental or South Pacific localities (advance shifts of 6.5 to 10 hours) were instructed to expose themselves to either bright white light (2000 lux) or dim red light (<100 lux) for two or 
three hours on awakening in the morning for three days. No differences were found between the group means for any sleep measure, but exposure to bright light early in the morning appeared to facilitate the consolidation of sleep into a single night-time episode. In the latter, ${ }^{56}$ four subjects were polysomnographically recorded before and after a flight from Tokyo to San Francisco (eight hour advance). In San Francisco, the subjects were requested to go bed at 2300 hours and wake up by 1000 hours and were exposed for three consecutive days to either bright (>3000 lux) or dim ( $<500$ lux) light for three hours starting at 1100 hours $(0300$ hours Tokyo time). The bright light treatment seemed to be effective in accelerating circadian reentrainment. However, the sample population of these studies is small, and the Consensus Report for Light Treatment for Sleep Disorders ${ }^{54}$ concluded that "much remains to be learned before procedures can be developed that are at once effective, reliable, and practical. For this to happen, optimal combinations of several light exposure parameters must be first defined and tailored to specific flight situations".

\section{Chronobiotic drugs}

Chronobiotic drugs are drugs that specifically affect some aspect of biological time structure, but they must be administered at the right time of the day. In the last few years, several putative chronobiotic drugs have been evaluated-for example, barbiturates, ${ }^{57}$ short acting benzodiazepines, ${ }^{58}$ serotonin-depleting agents, ${ }^{59}$ and corticosteroids, ${ }^{60}$ with unsatisfactory or inconclusive results. ${ }^{10}$

The pineal hormone melatonin ( $\mathrm{N}$-acetyl-5methoxytryptamine) is a very interesting chronomodulating substance. It is normally secreted at night, and in animals serves to transmit information about the light/dark cycle to the body. ${ }^{61}$ However, melatonin acts in a highly pleiotropic fashion, also eliciting secondary humoral immunological responses-for example, via interleukin- 4 and other cytokines, or acting as a powerful radical scavenger. ${ }^{62}$ The first reported administration of melatonin ${ }^{63}$ induced a mild sedative effect, which was initially considered to be a pharmacological side effect. In fact, the hypnotic effect of melatonin is considered to be an integral part of its physiological action. In an early study using polysomnography, ${ }^{64}$ intravenous infusion of melatonin before bedtime was associated with significantly increased sedation, reduced psychomotor activity, and shortened sleep onset latency. A recent study on healthy volunteers ${ }^{65}$ provided further evidence that nocturnal melatonin secretion may be involved in sleep onset and that exogenous melatonin may be useful in treating insomnia. Low oral doses of melatonin, just enough to increase serum melatonin concentrations to levels normally occurring at night, ${ }^{66}$ produce hypnotic effects when given in the evening. Moreover, in humans, melatonin is likely to be an effective hypnotic agent to combat sleep disruption associated with elevated temperature due to low circulating melatonin levels, and it may also improve sleep disruption caused by drugs that interfere with melatonin production-for example, $\beta$-blockers and benzodiazepines. ${ }^{67}$ Melatonin has been utilised for treatment of jet lag induced rhythm disturbances. In an early study reporting its use in transmeridian travellers, Arendt et $a l^{78}$ administered melatonin to 17 subjects over a period of 14 days after a westward flight from London to San Francisco. Compared with placebo, melatonin significantly reduced the negative feelings of jet lag and increased subjective alertness. Moreover, treated subjects also reported significantly reduced sleep onset latency and improved sleep quality, and endogenous melatonin and cortisol rhythms resynchronised more rapidly than in those consuming a placebo. In another study, ${ }^{69} 20$ volunteers with experience in transcontinental flights were pretreated with melatonin ( $5 \mathrm{mg}$, orally) for three days before the flight, during the flight, and for three days after arrival at their destination. The subjects pretreated with melatonin, compared with those taking placebo, reported significantly reduced feelings of jet lag and less time to establish a normal sleep pattern, reaching a "normal energy level" earlier than the controls and reporting lower levels of tiredness during the day. A controlled double blind trial with melatonin was performed in healthy volunteers flying between North America and France selected for their sensitivity to eastward jet lag. ${ }^{70}$ The protocol was simple, since one daily capsule of melatonin was administered on the day of return and the three consecutive evenings thereafter. A significant difference in self rating for global treatment efficacy, morning fatigue, and evening sleepiness was found in the melatonin compared with placebo group. Another study ${ }^{71}$ investigated the efficacy of oral melatonin in alleviating jet lag in a flight crew after a series of international flights and the optimal time for its consumption; it was shown that benefits are especially likely to occur when melatonin is consumed after arrival. Subjects taking melatonin for five days at home after the return flight showed a more rapid recovery of energy and alertness than those taking melatonin from one day before departure. It has been reported ${ }^{72}$ that the amount and the direction of the phase shift depends on the time of melatonin ingestion: evening administration advances the body clock and morning administration delays it.

\section{Conclusions}

Competitive athletes from many sport disciplines are nowadays, as never before, earning considerable sums of money by taking part in worldwide competitions. A growing series of events-for example, tournaments, international meetings, world series, world cup challenges, sponsored trophies-have greatly lengthened the competitive season. Only a few years ago, worldwide competitions were limited to the Olympic Games, World Championships and a few other "top events". Thus athletes are now required to travel rapidly all over the world, with no allowance for any "synchronisation" other than television network schedules. Both the next winter and summer Olympic Games 
(scheduled to take place in Japan and Australia respectively) will require the crossing of several time zones, especially by European teams.

As for measures for alleviating jet lag in athletes, preadaptation (because of social and behavioural constraints) is hardly practicable and also ineffective, and adaptation at the place of competition requires time and money. Appropriate timing and composition of meals may be useful for accelerating the adaptation of circadian rhythms to the timing of environmental synchronisers at the athlete's destination, although limitations set by commercial airlines and/or restrictions of the training diet have to be considered. Phototherapy is undoubtedly of interest, but still far too broad a practical standardisation. Probably, in the future, the combined use of bright light and melatonin ingestion will give satisfactory results, although further exhaustive studies specifically targeted to competitive athletes are needed. Although melatonin is not licensed for use in Europe, it is freely available in the United States in varying grades of purity, and most frequent flyers and/or top athletes regularly consume it. Unfortunately, the dosage and timing is not always correct, and it should be stressed that the hypnotic action of melatonin may have disappointing consequences on athletic performance. Thus it is advisable to consult specialists for personalised administration schedules.

This work was supported, in part, by grants from the Ministero della Università e della Ricerca Scientifica e Tecnologica (MURST) $60 \%$, Università di Ferrara. We thank Mrs Maria Cecilia Barbi, Librarian of the Institute of Internal Medicine, for her helpful collaboration.

1 Winget CM, DeRoshia CW, Markley CL, et al. A review of human physiology and performance changes associated
with desynchronisis of biological rhythms. Aviat Space Environ Med 1984;55:1085-96.

2 Graeber RC. Alterations in performance following rapid transmeridian flight. In: Brown FM, Graeber RC, eds. Rhythmic aspects of behaviour. Hillsdale, New Jersey: Lawrence Erlbaum Associates, 1982:173-212.

3 Lavernhe J, LaFontaine E, Pasquet J. Les réactions subjectives et objectives aux ruptures des rythmes circadiens lors des vols commerciaux long courriers est-ouest et vice-versa. vols commerciaux long courriers est-ouest et vice-
Revue de Medecine Aeronautic Spatiale 1968;7:121-3.

4 Preston FS. Further sleep problems in airline pilots on Preston FS. Further sleep problems in airline pilots on
worldwide schedules. Aerospace Medicine 1973;44:775-82.

5 Preston FS, Bateman SC, Short RV, et al. Effects of flying and of time changes on menstrual cycle length and performance in airline stewardesses. Aerospace Medicine 1973;44:438-43.

6 Suvanto S, Prtinen M, Harma M, et al. Flight attendants' desynchronosis after rapid time zone changes. Aviat Space Environ Med 1990;61:543-7.

7 Klein K, Wegmann H. The resynchronization of human circadian rhythms after transmeridian flights as a result of flight direction and mode of activity. In: Scheving LE, ed. Chronobiology. Tokyo: Igaku-Shoin, 1974:564-70.

8 Aschoff J, Hoffmann K, Pohl H, et al. Re-entrainment of circadian rhythms after phase-shifts to the zeitgeber. Chronobiologia $1975 ; 2: 23-78$.

9 Winget CM, Soliman MRI, Holley DC, et al. Chronobiology of physical performance and sports medicine. In: Toui-
tou Y, Haus E, eds. Biologic rhythms in clinical and laboratory tou Y, Haus E, eds. Biologic rhythms in clinical and laboratory
medicine. Berlin and Heidelberg: Springer Verlag, 1992: medicine.

10 Redfern P, Minors D, Waterhouse J. Circadian rhythms, jet lag, and chronobiotics: an overview. Chronobiol Int 1994;11: 253-65.

11 Moline ML, Pollack CP, Monk TH, et al. Age-related differences in recovery from simulated jet-lag. Sleep 1992;15:28 40.

12 Suvanto S, Härma M. The prediction of the adaptation of circadian rhythms to rapid time zone changes. Ergonomics 1993;36:111-6.

13 Minors D, Waterhouse J. Circadian rhythms and the human. London: Wright PSG, 1981.

14 Akerstedt T. Altered sleep/wake patterns and circadian rhythms. Acta Physiol Scand Suppl 1979;469:1-48.

15 Reilly T. Human circadian rhythm and exercise. Crit Rev Biomed Eng 1990;18:165-80.

16 Smolensky MH, Tatar SE, Bergman SA, et al. Circadian rhythmic aspects of human cardiovascular function: a review by chronobiologic statistical methods. Chronobiologi $1976 \cdot 3: 337-71$

17 Pickering TG. The influence of daily activity on ambulatory blood pressure. Am Heart f 1988;116:1141-5.

18 Portaluppi F, Vergnani L, Manfredini R, et al. Endocrine mechanisms of blood pressure rhythms. Ann N Y Acad Sci 1996;783:113-31.

19 Moore JG. Chronobiology of the gastrointestinal system. In: Touitou Y, Haus E, eds. Biological rhythms in clinical and laboratory medicine. Berlin: Springer-Verlag, 1992:410-17.

20 Goo RH, Moore JG, Greenberg E, et al. Circadian variation in gastric emptying of meals in man. Gastroenterology 1987; 93:513-18.

21 Touitou Y, Touitou C, Bogdan A, et al. Circadian and seasonal variations of electrolytes in ageing humans. Clin Chim Acta 1989;180:245-54.

22 Robertson WG, Hodgkinson A, Marshall DH. Seasonal variations in the composition of urine from normal subjects: a ations in the composition of urine from normal subje
longitudinal study. Clin Chim Acta 1977;80:347-53.

23 Winget CM, DeRoshia CW, Holley DC. Circadian rhythms and athletic performance. Med Sci Sports Exerc 1985;17: and athletic

24 Atkinson G, Reilly T. Circadian variation in sports performance. Sports Med 1996;21:292-312.

25 Kleitman N. Sleep and wakefulness. Chicago: University of Chicago Press, 1963

26 Gifford LS. Circadian variation in human flexibility and grip strength. Australian fournal of Physiotherapy 1987;33:3-9. 7 Coldwells A, Atkinson G, Reilly T. Sources of variation in back and leg dynamometry. Ergonomics 1993;37:79-86.

28 Atkinson G, Greeves J, Cable T, et al. Day-to-day and circadian variability of leg strength measured with the LIDO dian variability of leg strength measured with the
isokinetic dynamometer. $\mathcal{F}$ Sports Sci $1995 ; 13: 18-19$.

29 Hill DW, Borden DO, Darnaby KM, et al. Effect of time of day on aerobic and anaerobic responses to high intensity day on aerobic and anaerobic responses to
exercise. Can $\mathcal{F}$ Sports Sci 1992;17:316-19.

30 Reilly T, Baxter C. Influence of time of day on reactions to cycling at a fixed high intensity. Br $\mathcal{f}$ Sports Med $1983 \cdot 17: 128-30$

31 Wright V, Dawson D, Longfield MD. Joint stiffness: its characterisation and significance. Biological Medical Engineering 1969;4:8-14

32 Procacci P, della Corte M, Zoppi M, et al. Rhythmic changes of the cutaneous pain threshold in man. Chronobiologia 1974;1:77-96

33 Coldwells A, Atkinson G, Reilly $\mathrm{T}$, et al. Self-chosen work-rate determinates day-night differences in work capacity. Ergonomics 1993;36:313.

34 Atkinson G, Reilly T. Effects of age and time of day on preferred work rates during prolonged exercise. Chronobiol Int 1995;12:121-34.

35 Hildebrandt G, Gutenbrunner C, Reinhart C. Circadian variation of isometric strength training in man. In: Morgan $\mathrm{E}$, ed. Chronobiology and chronomedicine. Frankfurt: Peter Lang, 1990;2:322-9.

36 Folkard S, Monk TH, Bradbury R, et al. Time of day effects in school children's immediate and delayed recall of meaningful material. Br f Psychol 1977;68:45-50.

37 Reilly T, Down A. Circadian variation in the standing broad jump. Percept Mot Skills 1986;62:830.

38 Reilly $\mathrm{T}$, Down A. Investigation of circadian rhythms in anaerobic power and capacity of the legs. 7 Sports Med Phys Fitness 1992;33:343-7.

39 Reilly T, Marshall S. Circadian rhythms in power output on a swim bench. Fournal of Swimming Research 1991;7:11-13.

40 Sinnerton S, Reilly T. Effect of sleep loss and time of day in swimmers. In: Maclaren D, Reilly $\mathrm{T}$, Lees A, eds. London: E and FN Spon, 1992:399-405.

41 Monk TH, Leng VC. Time of day effects in simple repetitive tasks: some possible mechanisms. Acta Psychol (Amst) 1982;51:207-21.

42 Monk TH. Chronobiology of mental performance. In: Touitou Y, Haus E, eds. Biological rhythms in clinical and laboratory medicine. Berlin: Springer-Verlag, 1992:208-13.

43 Folkard S, Monk TH. Chronopsychology: circadian rhythms and human performance. In: Gale A, Edwards JA, eds. Attention and performance. New York: Academic Press, $1983 ; 2: 55-78$.

44 Klein KE, Bruner H, Wegmann HM, et al. Die Veränderung der psychomotorischen Leistungsbereitschaft als Folge pharmakodynamischer Einwirkung verschiedener Substanzen mit potentiell sedierenden Effekt. Arzneimittelforschung 1967;17:1048-51.

45 Jehue R, Street D, Huizenga R. Effect of time zone and game time changes on team performance: National game time changes on team performance: Natio

46 Recht LD, Lew RA, Schwartz WJ. Baseball teams beaten by jet lag. [Letter]. Nature 1995;377:583.

47 Sasaki T. Effect of jet lag on sports performance. In: Scheving LE, Halberg F, eds. Chronobiology: principles and applications to shifts in schedules. Rockville: Sijthoff and Noordhoff, 1980:417-34

48 Reilly T, Atkinson G, Budgett R. Effects of temazepam on physiological and performance variables following a westerly flight across five time zones. F Sports Sci 1997;15:62.

49 Minors DS, Waterhouse JM. Anchor sleep as a synchronizer of abnormal routine. Int f Chronobiol 1981;7:165-88.

50 Reilly T, Atkinson G, Waterhouse J. Biological rhythms and exercise. Oxford: Oxford University Press, 1997.

51 Reilly $\mathrm{T}$, Atkinson G, Waterhouse J. Travel fatigue and jet-lag. I Sports Sci 1997; 15:365-9.

52 Wurtman RJ. Nutrients that modify brain function. Sci Am 1982;246:50-9. 
53 Czeisler CA, Allan JS, Strogatz SH, et al. Bright light resets the human circadian pacemaker independent of the timing of the sleep-wake cycle. Science 1986;233:667-71.

54 Boulos Z, Campbell SC, Lewy AJ, et al. Light treatment for sleep disorders: consensus report. VII. Jet lag. $f$ Biol Rhythms 1995;10:167-76.

55 Cole RJ, Kripke DF. Amelioration of jet lag by bright light treatment: effects on sleep consolidation. Sleep Research 1989;18:411.

56 Sasaki M, Kurosaki Y, Onda M, et al. Effects of bright light on circadian rhythmicity and sleep after transmeridian flight. Sleep Research 1989;18:442.

57 Simpson HW. Chronobiotics: selected agents of potential value in jet lag and other dyschronisms. In: Scheving LE, Halberg F, eds. Chronobiology: principles and applications to shift and schedules. Rockville: Sijthoff and Noordhoff, 1980: 433-46.

58 Wee BE, Turek FW. Midazolam, a short acting benzodiazepine, resets the circadian clock of the hamster. Pharmacol Biochem Behav 1989;32:901-6.

59 Simpson HW, Bellamy N, Halberg F. Double blind trial of a possible chronobiotic (quiadon): field studies in NW Greenland. Int f Chronobiol 1973;1:287-311.

60 Christie GA, Moore-Robinson M. Project Pegasus: circadian rhythms and new aspects of corticosteroids. Clinical Trials fournal 1970;7:7-135.

61 Tamarkin L, Baird CJ, Almeida OFX. Melatonin: a co-ordinating signal for mammalian reproduction. Science 1985;227:714-20.
62 Hardeland R, Rodriguez C. Versatile melatonin: a pervasive molecule serves various functions in signaling and protecmolecule serves various functions in

63 Lerner AB, Case JD. Melatonin. Federation Proceedings 1960;19:590-2.

64 Cramer H, Rudolph J, Consbruch U. On the effects of melatonin on sleep and behavior in man. Adv Biochem Psychopharmacol 1974;2:187-91.

65 Zhdanova IV, Wurtman RJ, Lynch HJ, et al. Sleep-inducing effects of low doses of melatonin ingested in the evening. Clin Pharmacol Ther 1995;57:552-8.

66 Dollins AB, Zhdanova IV, Wurtman RJ, et al. Effect of inducing nocturnal serum melatonin concentrations in daytime on sleep, mood, body temperature, and performance. Proc Natl Acad Sci USA 1994;91:1824-8.

67 Dawson D, Encel N. Melatonin and sleep in humans. 7 Pineal Res 1993;15:1-12.

68 Arendt J, Aldhous M, English J, et al, The effects of jet-lag and their alleviation by melatonin. Ergonomics 1987;30: 1379-93.

69 Petrie K, Conaglen JV, Thompson L, et al. Effect of melatonin on jet lag after long haul flights. BMF 1990;298: 05-7.

70 Claustrat B, Brun J, David M, et al. Melatonin and jet-lag: confirmatory result using a simplified protocol. Biol Psychiatry 1992;32:705-11.

71 Petrie K, Dawson AG, Thompson L, et al. A double-blind trial of melatonin as a treatment for jet-lag in international cabin crew. Biol Psychiatry 1993;33:526-30.

72 Lewy AJ, Ahmed S, Jackson JM, et al. Melatonin shifts human circadian rhythms according to a phase response curve. Chronobiol Int 1992;9:380-93.

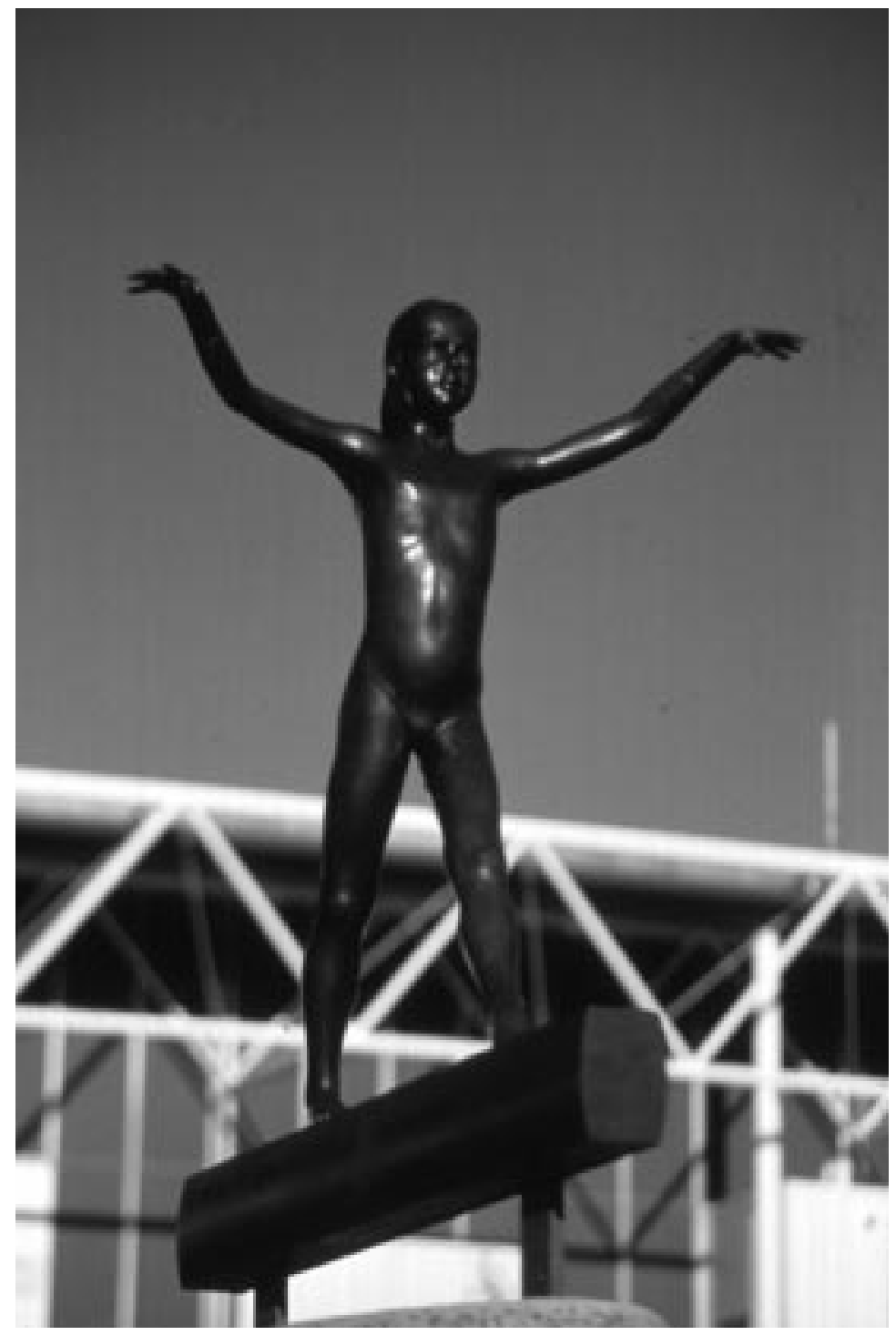

Australian Institute of Sport facilities gymnastics training hall, "The gymnast" by fohn Robinson. Reproduced with permission. 